\title{
Politikwissenschaft und Politikberatung: Reflektionen anlässlich der aktuellen Krise
}

\author{
Andreas Busch
}

Zusammenfassung: Der Beitrag diskutiert das Verhältnis von politikwissenschaftlicher Expertise und politischer Praxis vor allem am Beispiel der Entwicklung von Beratungsinstitutionen in der Bundesrepublik Deutschland und den USA unter besonderer Berücksichtigung der zeitgeschichtlichen Umstände. Es wird gezeigt, dass die Nachfrage und Wertschätzung wissenschaftlicher Beratung der Politik zyklischen Schwankungen unterliegt, wobei Gründe für das bisweilen schwierige Verhältnis sowohl auf Seiten der Wissenschaft als auch bei der Politik zu finden sind. Schließlich wird konstatiert, dass ein konstruktiver Dialog zwischen den gesellschaftlichen Teilsystemen Politik und Wissenschaft (und damit eine sinnvolle wissenschaftliche Politikberatung) auf substantielle Schwierigkeiten beispielsweise bei der Institutionalisierung stößt.

Schlüsselwörter: Politikwissenschaft · Politikberatung • Wirtschaftskrise · „Policy science“

\section{Political science and policy advice: reflections on the occasion of the current crisis}

\begin{abstract}
The article discusses the relationship between political science expertise and political practice by focusing on the development of institutionalized policy advice in the Federal Republic of Germany and the United States. Taking particular account of concrete historical contexts, we can show that demand for (and appreciation of) academic policy advice is subject to business cycles to which both political and academic factors contribute. The article concludes that a constructive dialogue between the societal sub-systems politics and academia (and thus meaningful academic policy advice) faces substantial problems, not least of institutionalization
\end{abstract}

Keywords: Political science $\cdot$ Policy advice $\cdot$ Economic crisis $\cdot$ Policy science

Online publiziert: 09.10 .2009
(C) Die Autor(en) 2009. Dieser Artikel ist auf Springerlink.com mit Open Access verfügbar.

Der vorliegende Beitrag ist eine leicht überarbeitete und mit Literaturnachweisen versehene Fassung des Vortrags, den der Autor am 3. Juli 2009 auf Einladung des Instituts für Politische Wissenschaft der Universität Heidelberg aus Anlass des 75. Geburtstags von Klaus von Beyme gehalten hat

Prof. Dr. A. Busch $(\bowtie)$

Seminar für Politikwissenschaft, Georg-August-Universität Göttingen

Platz der Göttinger Sieben 3, 37073 Göttingen, Deutschland

E-Mail: andreas.busch@sowi.uni-goettingen.de 


\section{KERNAUSSAGEN}

Die gegenwärtige Finanz- und Wirtschaftskrise wirft Fragen nach dem Wissen des Staates und der Rolle der akademischen Beratung auf. Die Weltwirtschaftskrise der 1930er Jahre war in den USA eine treibende Kraft bei der Etablierung akademischer Ressourcen zur Politikberatung, die auch auf das Fach zurückwirkte. Die Reformphase der 1960er Jahre brachte in den USA wie in der Bundesrepublik Hoffnung auf eine „Verwissenschaftlichung von Politik“, die jedoch auf Probleme der Implementation stieß und ab den 1970er Jahren einer Ernüchterung Platz machte. Sowohl Politik als auch Wissenschaft haben bei der Politikberatung eigene Interessen, die eine konstruktive Kooperation erschweren.

\section{Die Krise, mangelndes Wissen und die Rolle des Staates}

Das Jahr 2009 scheint das Jahr runder Geburtstage und runder Jubiläen zu sein. Die Verfassung unseres Gemeinwesens, das Grundgesetz, ist in diesem Jahr 60 Jahre alt geworden. Und vor Klaus von Beymes Geburtstag haben wir in den letzten Monaten bereits runde Geburtstage von Ralf Dahrendorf und Jürgen Habermas gefeiert. Dieses Jahr so zu sehen ist aber natürlich die Perspektive eines Politikwissenschaftlers und nicht unbedingt repräsentativ für die Rezeption in der allgemeinen Bevölkerung. Die Chancen stehen nämlich gut, dass das Jahr 2009 in der allgemeinen Wahrnehmung eher als das Jahr der großen ökonomischen Krise in die Erinnerung eingehen wird.

Zwar hat die gegenwärtige Finanz- und Wirtschaftskrise bereits im Sommer 2007 begonnen und daher bereits ihren zweiten Geburtstag gefeiert. Aber erst mit dem Zusammenbruch der Bank Lehman Brothers im September 2008 ist die Krise tatsächlich in das allgemeine Bewusstsein getreten - und in den Mittelpunkt des politischen Handelns. Seit Herbst letzten Jahres sehen wir eine massive und sich beständig beschleunigende Vertiefung der Krise. Man kann das vielleicht am besten anhand einiger Zahlen illustrieren, die zeigen, wie sich die Erwartungen hinsichtlich der zukünftigen Entwicklung in den letzten Monaten verändert - und beständig verschlechtert - haben:

Noch im Frühjahr 2008 hatte die OECD für Deutschland im Jahr 2009 eine Wirtschaftswachstumsrate von 1,6\% vorhergesagt. Das war natürlich, bevor sich die Finanzkrise im Herbst dramatisch verschärfte! Im November 2008 reduzierte der Sachverständigenrat die Erwartungen dann bereits auf ein Nullwachstum. Ende Januar 2009 prognostizierte die Bundesregierung dann im Jahreswirtschaftsbericht, dass die deutsche Wirtschaft im laufenden Jahr um 2,25\% schrumpfen werde. Und die Frühjahrsprognose der OECD übertraf diese bereits düstere Prognose noch weiter: Im April 2009 sagte die OECD voraus, dass die Wirtschaftsleistung in Deutschland in diesem Jahr um 5,3\% zurückgehen werde. Wenn man sich vor Augen hält, dass der bisher schwerste Wachstumseinbruch in der Geschichte der Bundesrepublik - im Krisenjahr 1975 - einen Rückgang des BIP um $0,9 \%$ brachte, dann kann einem bei der Aussicht, nun mehr als das Fünffache zu erwarten, ja schon etwas schwindelig werden...

Doch nicht nur bei der Entwicklung und Vorhersage des Wirtschaftswachstums scheint es Schwierigkeiten zu geben. Ganz Ähnliches können wir bei den Prognosen hinsichtlich der Verluste der aktuellen Finanzkrise sehen. Gegen Ende des Jahres 2007 schätzte der Vorsitzende der amerikanischen Zentralbank, Ben Bernanke, dass sich die Verluste auf 
mehrere hundert Milliarden US Dollar, sicher aber weniger als eine halbe Billion Dollar belaufen würden. Ein Jahr später, im Januar 2009, schätzte der Chef des Internationalen Währungsfonds, Dominique Strauss-Kahn, dass die endgültige Summe der Verluste etwa beim Drei- bis Vierfachen der von Bernanke genannten Summen liegen werde - also bei 1,5 bis 2 Billionen US-Dollar. Und nur wenige Monate später, im April 2009, korrigierte der Währungsfonds diese Zahlen noch einmal beträchtlich nach oben, nämlich auf 4 Billionen Dollar. So Schwindel erregend diese Zahl - 4000 Milliarden Dollar! - klingen mag, so erfasst sie doch nur einen Teil der tatsächlich durch die Krise ausgelösten Verluste, nämlich die direkten Abschreibungen. Der Gesamtverlust an Vermögenswerten ist wohl noch viel höher einzuschätzen: Eine Studie der Asiatischen Entwicklungsbank schätzt die weltweit vernichteten Vermögenswerte auf 50 Billionen Dollar - und allein in Asien auf 9,6 Billionen Dollar, was dem Bruttoinlandsprodukt eines ganzen Jahres entspricht.

Was wir bei aller Unsicherheit aber sagen können, ist Folgendes: Die massive Krise der Finanzmärkte und die allgemeine Weltwirtschaftskrise, die sich daraus ergeben hat, haben weltweit den Staat wieder in eine völlig neue Rolle gebracht. In der Diskussion des letzten Jahrzehnts war der Staat ja schon weitgehend abgeschrieben - man betrachtete ihn weniger als Lösung denn als Ursache vieler Probleme. Grund dafür waren Prozesse der Globalisierung, der Privatisierung im Inneren und der Abgabe zahlreicher Kompetenzen nach außen. Viele sahen den Staat dadurch der Erosion und dem Niedergang anheim gegeben, und manche schrieben sogar schon sein Ende herbei. Doch in der Krise, die durch die vor kurzem noch gefeierte globalisierte Wirtschaft mit ihren hochkomplexen Finanzprodukten und ihrer internationalen Verflechtung von Amerika ausgehend weite Teile der Welt ergriffen hat, erlebt der Staat nun plötzlich eine Art Auferstehung. Gewissermaßen als Residualakteur muss er nun überall eingreifen, wo Not am Mann ist und Märkte sich selbst nicht helfen können - von Ausfallbürgschaften für Finanzunternehmen bis zu Hilfen für Autohersteller.

Wie der Staat mit dieser Herausforderung umgeht, wie er an sie heran geht und welche Erfolge und Misserfolge sich dabei ergeben, wird für die Politikwissenschaft (und insbesondere die vergleichende Staatstätigkeitsforschung) in den nächsten Jahren ein faszinierendes Arbeitsfeld darstellen. Denn wieder einmal haben wir - ähnlich wie Mitte der 1970er Jahre - einen Schock, der alle Länder in weitgehend gleicher Weise erfasst. Vom methodologischen Standpunkt aus ist das ein Quasi-Experiment, und es wird uns erlauben, die Reaktionen vieler Länder auf eine ähnliche Herausforderung zu untersuchen. Ich erwarte, dass wir hier auch weiterhin gravierende Unterschiede feststellen werden, die dem Einfluss national spezifischer Faktoren geschuldet sind.

Aber keine Sorge: Über Globalisierung ,and all that" möchte ich heute nicht sprechen. Ich möchte das eben Angeführte vielmehr zum Anlass nehmen, mich mit der Frage des Zusammenhangs zwischen Politik und Wissen zu beschäftigen. Der Staat - das macht die gegenwärtige Krise deutlich - kann der Verantwortung für den Umgang mit den Folgen der aktuellen Krise nicht ausweichen; ich habe ihn deshalb gerade den „Residualakteur“ genannt, der eingreifen muss, wenn die anderen Akteure - die Märkte, die großen Unternehmen und Banken, die sich noch vor kurzem von aller staatlichen Oberaufsicht etwa im Bereich der Finanzmärkte lossagen wollten - die Segel streichen.

Woher aber soll der Staat wissen, was zu tun ist? Woher soll er das Spezialwissen nehmen, das man etwa braucht, um etwa ein vom Zusammenbruch bedrohtes Bankensystem 
zu retten? Wie soll er wissen, welcher Eingriff am besten wirtschaftlichen Niedergang abfedert und übermäßige soziale Härten vermeidet? Der Staat handelt - zumal in der akuten Krise - also unter Bedingungen von massiver Unsicherheit über die Folgen seines Handelns.

Diese Unsicherheit befördert die politische Auseinandersetzung. Der amerikanische Politikwissenschaftler Hugh Heclo hat in seinem bekannten Buch über „Modern social politics in Britain and Sweden“ im Jahr 1974 deshalb Unsicherheit (neben Macht) als konstitutive Quelle von Politik herausgestellt:

Politics find its sources not only in power but also in uncertainty - men collectively wondering what to do. [...] Governments not only 'power' (or whatever the verb form of that approach might be); they also puzzle. Policy-making is a form of collective puzzlement on society's behalf; it entails both deciding and knowing. (Heclo 1974, S. 305)

In welchem Maße und mit welcher Gründlichkeit man sich verfügbares Wissen zu erschließen versucht, bevor politische Entscheidungen getroffen werden, hängt natürlich von der konkreten Situation ab. Einem Aufsatz des mittlerweile in Passau lehrenden Historikers Hans-Christof Kraus können wir beispielsweise entnehmen, dass vor der Gründung der Berliner Universität im Jahr 1807 der mit der Organisation beauftragte Kabinettsrat Carl Friedrich von Beyme seine Tätigkeit damit begann, ,von einer ganzen Reihe im Umfeld der preußischen Regierung ansässiger Gelehrter [...] Memoranden über eine in der preußischen Hauptstadt zu errichtende Universität" anzufordern (Kraus 2004, S. 64). Unter denen, die sich äußerten, waren so bedeutende Wissenschaftler wie der Theologe Friedrich Schleiermacher, der Philologe Friedrich August Wolf und der Philosoph Johann Gottlieb Fichte. Ihre Denkschriften wurden berühmt und haben zum Erfolg der Berliner Gründung wesentlich beigetragen. Heute wünscht man sich vielleicht, die damalige Bildungsministerin Edelgard Bulmahn (oder einer ihrer Beamten) hätte Ähnliches getan, bevor sie das deutsche Hochschulwesen in das Prokrustesbett des BolognaProzesses pressten - vielleicht wäre uns der eine oder andere Unsinn (und mit ihm die jüngsten Proteste) dann erspart geblieben!

Denn im Fall der Bulmahn'schen Reform fand das staatliche Handeln ja ohne großen Zeitdruck statt. Den Luxus hat Politik in der heutigen Krise nicht. Krisen haben immer den Bedarf nach Wissen und Beratung erhöht, weshalb sich einige der bekanntesten Institutionen der Politikanalyse auf Krisen zurückführen lassen. Aber Politikberatung ist nicht nur technisches Wissen, sie ist auch Handeln im politischen Raum und deshalb umstritten. Die gegenwärtige Krise muss deshalb auch zu Reflexionen über die Rolle von Wissenschaft bei der Politikberatung führen. Es ist wohl kein Zufall, dass die Ökonomen - in den Vereinigten Staaten wie auch in Deutschland - gerade eine intensive Debatte über die Grundlagen ihres Faches führen, unter anderem über ihre Rationalitätsannahmen. ${ }^{1}$ Auch der Politikwissenschaft würde es gut tun, sich Gedanken über die Möglichkeiten und Grenzen besserer Beratung von Politik zu machen, damit Krisen wie die gegenwärtige

1 Freilich ist es eine deutsche Besonderheit, diese Debatte im Zusammenhang mit der Besetzung von Lehrstühlen an der Universität Köln und hauptsächlich auf den Seiten des Handelblatts und der $F A Z$ zu führen - das Buch von Akerlof und Shiller (2009) bietet da tiefere Reflexionen. 
in Zukunft vielleicht vermieden werden können. Ich möchte heute einen Beitrag zu einer solchen Debatte leisten, indem ich einen reflektierenden Streifzug durch die Entwicklung der Politikberatung und der Rückwirkung auf unser Fach unternehme. Dabei möchte ich in drei Schritten vorgehen:

- Zunächst möchte ich kurz die Frage des Einflusses von Experten in der Politik diskutieren, und dann

- darlegen, wie, wann und wo sich die Politikwissenschaft in den letzten Jahrzehnten auf die politische Wirklichkeit beratend eingelassen hat, und welche Konjunkturzyklen es hier gegeben hat - also das Auf und Ab der Wichtigkeit, die man diesem Thema zugemessen hat.

- In einem dritten Schritt möchte ich deshalb über einige Gründe für die Schwierigkeiten im Verhältnis zwischen Öffentlichkeit, Wissenschaft und Politik reflektieren, bevor ich mit einigen theoretischen Überlegungen meinen Vortrag beenden werde.

\section{Theoretische Reflexionen und frühe Formen von Politikberatung}

In verfahrenen politischen Situationen, in denen es Schwierigkeiten bei der Mehrheitsfindung gibt, werden bisweilen so genannte „Expertenregierungen“ eingesetzt - das ist dann im wahren Sinne des Wortes eine „Expertokratie“. Vor zehn Jahren gab es eine Reihe solcher Regierungen in Italien, und in diesem Jahr wurde in Ungarn eine Expertenregierung eingesetzt. Doch abgesehen von demokratischen Ausnahmesituationen kann eine Präferenz für Expertenregierungen auch Merkmal einer bestimmten Art politischer Kultur sein - einer politischen Kultur, die konfliktscheu ist und Auseinandersetzungen mit dem Appell an das überlegene Wissen der Experten zu entgehen versucht. Solche Einstellungen können aus obrigkeitsstaatlichem Denken entstehen - Sie kennen bestimmt den Ausspruch von Kaiser Wilhelm II: „Ich kenne keine Parteien mehr, ich kenne nur noch Deutsche“. Insinuiert wird, dass Parteienstreit überflüssig ist und man sich doch einfach auf die bestmögliche Lösung einigen solle. Dass gerade die politische Kultur in Deutschland eine ist, die Partizipation bisweilen als Last (und Konflikt als illegitim) betrachtet, hat vor vielen Jahrzehnten Ralf Dahrendorf in seinem berühmten Buch „Gesellschaft und Demokratie in Deutschland“ herausgearbeitet. Dahrendorf argumentierte Mitte der 1960er Jahre, dass sich darin in Deutschland eine Fremdheit mit der Essenz der liberalen Demokratie zeige, die nun einmal auf dem regelgemäßen Austragen von Konflikten beruhe. Er schrieb damals:

Wo immer widersprüchliche Interessen in der deutschen Gesellschaft aufeinanderprallen, besteht die Tendenz, autoritäre und inhaltliche statt versuchsweiser und formaler Lösungen zu suchen. (Dahrendorf 1965, S. 163)

Man neige dazu, politische Probleme zu Rechtsfragen zu machen, Probleme an Experten zu delegieren und Konflikte zu scheuen (Dahrendorf 1965, S. 269).

Der Appell an das überlegene Wissen kann also zum einen ein Zeichen demokratischer Unreife sein. Zum anderen kann aber auch die Produktion von Wissen für Zwecke der Politikberatung abgelehnt werden, weil damit Hilfestellung für die Macht geleistet wird. 
Das Verhältnis zwischen Geist und Macht ist ja in Deutschland ein berühmt kompliziertes, und auch im Bereich der Politikberatung können wir das sehen. Das Wissenschaftszentrum Berlin für Sozialforschung - eine Institution, mit der Klaus von Beyme seit langem eng verbunden ist - feiert in diesem Jahr ja ebenfalls ein rundes Jubiläum, nämlich sein 40jähriges Bestehen. Es wurde damals auf Initiative mehrerer Bundestagsabgeordneter gegründet, um mit seiner Arbeit dem Parlament Hilfestellung bei seinen Entscheidungen zu leisten. Doch dieser Versuch, parlamentarische Entscheidungen damit auf eine rationalere Grundlage zu stellen, traf auf zum Teil erheblichen Widerstand.

Paul Stoop, der Leiter der Presseabteilung des WZB, hat unlängst in einem Artikel die Proteste geschildert, die beinahe die Gründung des Wissenschaftszentrums zum Scheitern gebracht hätten (Stoop 2009). Die westberliner Universitätspräsidenten, die Westdeutsche Rektorenkonferenz, die Gewerkschaft Erziehung und Wissenschaft und viele andere mehr formulierten massive Kritik an der geplanten öffentlichen Finanzierung einer privatrechtlichen Einrichtung. Die „Roten Zellen“ nannten das WZB eine „Ausbeuter- und Kriegstreiber-GmbH“ und einen „Technokraten-Olymp für die Mandarine der Zukunft“. Stephan Leibfried, heute Professor für Politikwissenschaft in Bremen und Sprecher eines Sonderforschungsbereiches zum Thema „Staatlichkeit im Wandel“, wetterte in der Frankfurter Rundschau vom 24. April 1970 gegen die ,clandestine Etablierung einer postuniversitären Funktionärselite-Schule“".

Solche Skrupel über das Verhältnis zwischen Geist und Macht waren den ersten Autoren der Gattung Politikberatung mehrere Jahrhunderte zuvor unbekannt. Ich rede hier von den Autoren der sogenannten „Fürstenspiegel-Literatur“, die im ausgehenden Mittelalter Schriften mit dem Ziel einer Theorie gerechter Herrschaftsausübung verfassten. Diese Schriften richteten sich an die weltlichen Fürsten, gaben ihnen Anweisungen zu guter Amtsführung, zur besten Regierungsweise (damals hieß das noch nicht ,good governance“!) und machten sie mit den Grundsätzen politischen Verhaltens vertraut. Vor allem während der Renaissance erlebte diese Gattung eine Blütezeit.

Waren die Schriften der Fürstenspiegel-Literatur vor allem normativ ausgerichtet, so fand im frühen 16. Jahrhundert eine radikale Neuorientierung statt. Damals veröffentlichte Niccolò Machiavelli sein berühmtes Buch „Der Fürst““ - „Il Principe“. Machiavelli nahm mit diesem Buch, mit dem er dem neuen Herrscher von Florenz, Lorenzo de Medici, Ratschläge für seine Herrschaft geben wollte, eine radikale Umorientierung vor und etablierte die Politik als ein eigenständiges Feld jenseits von Moral und Tugend (vgl. Münkler 1982). Für den Herrscher sollte es nun nicht mehr darum gehen, tugendhaft zu sein, sondern sich erfolgsorientiert zu verhalten. Er betrachte die Dinge „wie sie sind“ und nicht ,wie sie sein sollen“ - so schreibt Machiavelli selbst. Es steht also hier zum ersten Mal nicht mehr das normative, sondern das tatsächliche Handeln der Menschen im Vordergrund der Analyse. Vielen gilt Machiavelli deshalb als Erfinder des politischen Realismus, aber auch als ein Apologet der Machttechnik, dessen Schriften lange Zeit hoch umstritten waren. Noch heute wird das Adjektiv „machiavellistisch“ verwendet, wenn eine Verhaltensweise nur auf Gewinnung oder Erhaltung von Macht ausgerichtet erscheint.

Die Lehre von der Politik begann sich damals auch akademisch zu etablieren. Parallel zur Entstehung der modernen Staaten entstanden die Kameralwissenschaft als die Lehre von den fürstlichen Finanzen, die Ökonomik sowohl als Haus- wie auch als Staats- 
wirtschaftslehre und ein ganzer Kanon von technologisch orientierten Fächern wie die Landwirtschaftslehre, die Forstwissenschaft und der Bergbau. Wilhelm Bleek hat diese Entwicklung in seiner „Geschichte der Politikwissenschaft in Deutschland“ sehr detailliert nachgezeichnet (Bleek 2001). Es gab auch eine „Policeywissenschaft“ als das Fach von der Organisation und den Inhalten der inneren Verwaltung des Staatswesens. Das damalige Verständnis von „Policey“ war viel umfassender als der moderne Polizeibegriff, der sich heute auf die Erhaltung von Sicherheit und Ordnung beschränkt. ${ }^{2}$ „Policey“ war als Lehre von der guten Verwaltung dem englischen Begriff ,policy“ sehr eng verwandt - und den hier erläutern zu wollen hieße wahrscheinlich, Eulen nach Heidelberg zu tragen!

Ab dem späten 18. Jahrhundert bürgerte sich für dieses Konglomerat von Wissenschaften der Begriff der „Staatswissenschaften“ ein. Zu ihnen gehörten auch das Öffentliche Recht und die Statistik als Lehre von den Zuständen der Staaten sowie die Staatengeschichte. Vertreter dieser Staatswissenschaften waren in der ersten Hälfte des 19. Jahrhunderts, im so genannten Vormärz, Wortführer der liberal konstitutionellen Bewegung des Bildungsbürgertums. Als Neu-Göttinger muss ich in diesem Zusammenhang natürlich auf die „Göttinger Sieben“ und insbesondere auf Friedrich Christoph Dahlmann verweisen, die hier eine wichtige Rolle spielten. Doch nach dem Scheitern der Reformbemühungen der deutschen verfassungsgebenden Nationalversammlung von 1848/49 sank auch das Ansehen der Staatswissenschaften.

\section{Moderne Entwicklungen und die Konjunkturzyklen des Themas}

In den demokratischen Nationalstaaten, unter denen Deutschland ja bekanntlich ein Nachzügler war, etablierte sich die Politikwissenschaft gewissermaßen zwischen Geschichtsund Rechtswissenschaft. Die Schriften der damals führenden, ersten Vertreter des Faches Politikwissenschaft waren sehr stark institutionenorientiert. Liest man Bücher wie die von James Bryce („Modern Democracies“, 1921), Herman Finer („Theory and Practice of Modern Government“, 1932) oder Carl Joachim Friedrich („Constitutional Government and Democracy“, 1937), so fällt auf, dass es sich hierbei fast ausschließlich um Institutionenbeschreibungen handelt. Die damalige Politikwissenschaft hatte noch kein abstraktes Verständnis vom politischen Prozess und noch weniger von politischen Inhalten - dem also, was wir heute als ,,policy“ bezeichnen. Und man hatte damals noch nicht das Konzept eines ,politischen Systems“ entwickelt, innerhalb dessen man Institutionen, Prozesse und Inhalte von Politik aufeinander beziehen kann.

Aber nicht nur die wissenschaftsimmanente Entwicklung, auch die Bedürfnisse des Staates beziehungsweise der Regierung haben einen Einfluss auf die Entwicklung des Verhältnisses zwischen Politikwissenschaft und politischer Praxis gehabt. Genaues Wissen um den Politikprozess und seine Eigenschaften ist ja vor allem wichtig für Regierungen und Politiker, die an den bestehenden Verhältnissen etwas ändern wollen. Zielt eine Regierung hingegen lediglich auf Verwaltung und Bewahrung des Bestehenden, so wird sie kaum einen erhöhten Bedarf an Information und Beratung über Politik haben.

2 Hierzu unlängst zusammenfassend von Beyme 2009. 
Im Zuge der Demokratisierung zu Beginn des 20. Jahrhunderts und dem Übergang von einem eher passiven zu einem immer aktiver handelnden Staat ist deshalb auch der Bedarf an Politikwissen und an Politikberatung gestiegen. Dies war zunächst der Fall in den Vereinigten Staaten von Amerika, wo bereits zu Beginn des 20. Jahrhunderts (in der so genannten Progressive Era) reformorientierte Politiker nach Experten suchten, deren Wissen sie für die Entscheidungen von Regierung und Parlament nutzen konnten. Politische Entscheidungen sollten auf diese Weise auf eine rationalere Grundlage gestellt werden und auch zu einem gewissen Maß dem Parteienstreit entzogen werden.

Wir können also das, was wir im Rückblick als die ,policy science“ bezeichnen d. h. die konkrete, auf Inhalte von Politik orientierte Ausrichtung der Politikwissenschaft und ihrer praktischen Anwendung - als ein Ergebnis eines Prozesses von Angebot und Nachfrage begreifen. In den 1930er Jahren waren es vor allem die damalige Weltwirtschaftskrise und die „Great Depression“, die eine massiv gestiegene Nachfrage nach wissenschaftlichem Politikwissen entfalteten. Präsident Roosevelt versammelte einen „brains trust“" von Sozialwissenschaftlern um sich, um die Politik des „New Deal“" zu konzipieren und umzusetzen. Zur gleichen Zeit - im Jahr 1936 - entstand an der Harvard University durch eine Stiftung die Graduate School of Public Administration, die sich spezifisch mit den Aufgaben staatlicher Politik befassen und daneben auch Politikexperten ausbilden sollte, die dann der staatlichen Verwaltung helfen könnten, die Reformpolitik der Roosevelt-Regierung umzusetzen. Hier entstand also zum ersten Mal eine akademische Ausbildungsstätte, deren Ziel die Praxis und die Beeinflussung der Praxis war - zum einen durch praxisorientierte Forschung und zum anderen durch die Ausbildung von Experten für die Praxis. Man konnte an dieser Institution Studiengänge zu Themen wie ,,public policy“, staatliche Verwaltung, Stadtplanung oder später auch internationale Entwicklung belegen.

Die in diesen Studiengängen akademisch trainierten Fachleute - Wirtschaftswissenschaftler, Politikwissenschaftler, Psychologen etc. - waren dann eine große Hilfe für die Vereinigten Staaten nach ihrem Eintritt in den Zweiten Weltkrieg. Viele Experten trugen zum Sieg in diesem Krieg bei, und dass es eine Vielzahl von sehr gut durchdachten Plänen für die Nachkriegssituation gab, verdankt sich ebenfalls dem Einsatz dieser Politikexperten. Nach dem Zweiten Weltkrieg kam es dann zur Gründung einer Reihe von Beratungsinstitutionen - etwa der National Science Foundation oder des Council of Economic Advisers. Auch erste Institutionen, die vertragsgebundene Politikforschung vornehmen konnten, entstanden - etwa (vor allem im Verteidigungssektor) die RAND Corporation und (etwas allgemeiner positioniert) die Brookings Institution.

Gegen Ende der 1940er Jahre bekam der neue Ansatz dann auch eine theoretische Fundierung und einen Namen: ,policy science“. Die Entstehung dieses Konzeptes ist vor allem mit dem Namen des amerikanischen Politikwissenschaftlers Harold Lasswell verbunden, der lange an der Yale University lehrte. Lasswell hatte während des Krieges die Nazipropaganda analysiert und die amerikanische Regierung beraten. Er hatte in den 1930er Jahren das Abgleiten vieler europäischer Staaten in autoritäre und totalitäre Regierungsformen mit Sorge beobachtet und wollte nun einen Beitrag dazu leisten, Politik in der Demokratie zu verbessern. Das Ziel war, so Lasswell, die demokratische Politik durch mehr Wissen zu verbessern und dadurch die Demokratie zu stabilisieren. Er nannte sein Konzept deshalb auch in seinem berühmten Buch von 1951 die ,policy sciences of 
democracy“ (Lerner u. Lasswell 1951). Der Ansatz der neuen politikwissenschaftlichen Analyserichtung - und sicher der Ansatz von Lasswell - war also keineswegs technokratisch oder wertfrei. Wir können ihn vielleicht am besten als einen besorgten Paternalismus beschreiben, denn Lasswell machte sich vor allem Sorgen, dass die einzelnen Bürger ihre wirklichen, langfristigen Interessen selbst nicht gut einschätzen könnten, da ihnen dazu das Wissen fehle. Er war davon überzeugt, dass eine auf Rationalität basierende Politik die beste Grundlage für ein stabiles, wohlhabendes und friedliches Gemeinwesen sein werde, und er hoffte, dass das von ihm mitentwickelte Konzept der „policy science“ dazu einen Beitrag werde leisten können.

$\mathrm{Zu}$ den wichtigsten heuristischen Neuerungen, die Lasswell in die Politikwissenschaft einführte, zählte das Konzept des ,ppolicy cycle“ - des Politikzyklus. Dieses Konzept erlaubte es, inhaltliche Politik nun nach verschiedenen Phasen analytisch zu unterscheiden und zu zerteilen. Man konnte sich dann Gedanken machen, mit welchen Methoden und Werkzeugen man in den verschiedenen Phasen der Politik am besten eingreifen konnte. Aus dieser Perspektive gesehen handelt es sich bei Politik vor allem um das Lösen von Problemen. Als Ausgangspunkt des ,,policy cycle“ wird zunächst das Problem genau definiert, bevor man sich daran macht, alternative Lösungsmöglichkeiten herauszuarbeiten. Diese Handlungsalternativen werden dann gegeneinander abgewogen - und in einem politischen Prozess wird die bestmögliche Lösung ausgewählt. Nach dieser Entscheidung geht es in die Phase der Implementation - oder Umsetzung der Politik. Und nachdem die Politik umgesetzt worden ist, folgt eine weitere Phase, in der ihre Ergebnisse evaluiert werden. Abhängig davon, ob die Evaluierung positiv oder negativ ausfällt, kommt es danach entweder zur Terminierung (also zur Beendigung) der Politik, oder es wird eine Novellierung der Politik angestrebt, in der die ursprünglichen Defizite durch eine Reform der Politik behoben werden sollen.

Die Perspektive, Politik als Mittel zur Lösung vorgefundener Probleme anzusehen, stieß vor allem in den 1960er Jahren auf große Resonanz. Dies war in verschiedenen Ländern der Fall, aber zuerst vor allem in den Vereinigten Staaten von Amerika. Die neue Kennedy-Administration sah sich einer Reihe von wichtigen Herausforderungen gegenüber - unter ihnen der so genannte „Sputnik-Schock“ der späten 1950er Jahre, der die USA an ihrer technologisch-wirtschaftlichen Überlegenheit gegenüber der Sowjetunion hatte zweifeln lassen. Die bipolare Konfrontation der Nachkriegszeit war damals auf einem Höhepunkt, und neben dieser existenziellen Bedrohung plante die neue Regierung auch noch umfassende sozialpolitische Reformen, die unter dem Stichwort der „Great Society" liefen.

Würde man diese anspruchsvolle Agenda umsetzen - und damit die Vereinigten Staaten von Amerika substantiell verändern - können? Die damalige Atmosphäre war in dieser Hinsicht sehr optimistisch. Die neue Regierung nahm sich vor, die „besten und klügsten“ des ganzen Landes in der Regierung zu versammeln und die modernsten Methoden anzuwenden, um ihre Ziele zu erreichen. Der neue Präsident rekrutierte für seine Regierung führende Akademiker der Zeit wie den Dekan der Faculty of Arts and Sciences an der Harvard University, McGeorge Bundy, und den Ökonomen John Galbraith, von der RAND Corporation den Verteidigungsspezialisten Charles Hitch und von der Spitze der amerikanischen Industrie den Chef der Ford Motor Company, Robert McNamara. Die damals dominante Vorstellung war, dass man sich zwar großen Herausforderungen gegenüber 
sehe, diese aber durch die Nutzung moderner Methoden und den Einsatz von Expertenwissen werde lösen können. Präsident Kennedy legte dies im Detail in seiner Rede an der Yale University im Jahr 1962 dar, in der er ausführte, dass die meisten Probleme des Landes technische und Verwaltungsprobleme sein. Zu ihrer Lösung sei sehr komplexes Wissen erforderlich, und die Entscheidungen erforderten deshalb den Einsatz von Experten, da das zur Lösung der Probleme notwendige Wissen jenseits der Fähigkeiten der meisten Bürger sei. Deshalb müssten moderne Entscheidungen jenseits der Aufgeregtheiten und Leidenschaftlichkeiten des normalen politischen Systems getroffen werden.

$\mathrm{Zu}$ den neuen Mitteln, die man zunächst im Verteidigungsministerium und dann generell im Politikprozess einsetzte, gehörte das so genannte „Planning Programming Budgeting System“ oder PPBS, das erstmals eine Kosten-Nutzen-Analyse auf politische Programmentscheidungen und Budgetanschaffungen anwandte. Dies erwies sich als sehr erfolgreich zur Eindämmung der Kosten, die in der Vergangenheit sehr häufig über das geplante Ziel hinaus geschossen waren. Mit der Verbreitung solcher Techniken im politischen und im Verwaltungsapparat stieg die Nachfrage nach Graduierten, die mit diesen Techniken umgehen konnten. Als Folge entstand an den führenden amerikanischen Universitäten ab Mitte der 1960er Jahre eine Vielzahl von Graduiertenprogrammen in ,public policy“, so etwa in Michigan, in Berkeley, an der Carnegie-Mellon University und an der University of Pennsylvania. Der Trend zur „Verwissenschaftlichung von Politik“ verbreitete sich von den Vereinigten Staaten ausgehend auch auf viele andere Länder.

Auch in der Bundesrepublik Deutschland strebte man in den 1960er Jahren eine ,Verwissenschaftlichung von Politik“ an. In den damaligen Debatten über „Steuerung“ und „Futurologie“ spielte die Politikwissenschaft als akademisches Fach jedoch kaum eine Rolle - sie war auf „Demokratiewissenschaft“ fokussiert und hatte die modernen Konzepte der ,,policy sciences“ noch nicht breiter rezipiert. Die Führung bei der Etablierung von Politikberatung in der Bundesrepublik Deutschland lag in den selben Sektoren wie in den Vereinigten Staaten von Amerika: zum einen im Bereich der Sicherheitspolitik und zum anderen im Bereich der Wirtschaftspolitik. Im Bereich der Sicherheitspolitik wurde 1962 auf private Initiative hin die „Stiftung Wissenschaft und Politik“ gegründet, der 1965 auf Beschluss des Deutschen Bundestages die Bundesrepublik Deutschland als Gesellschafter beitrat. Mehrere Dutzend Wissenschaftler beschäftigten sich im Forschungsinstitut der Stiftung mit internationaler Politik, nach Weltregionen gegliedert. Sie saßen im beschaulichen Ebenhausen, 20 Kilometer südlich von München - böse Zungen behaupten, bei der Wahl des Standortes habe vor allem die Nähe zum Bundesnachrichtendienst im benachbarten Pullach Pate gestanden.

Nur wenige Kilometer entfernt von Ebenhausen, am Starnberger See, entstand 1970 ein weiteres Institut, das seine Existenz der Planungseuphorie der 1960er Jahre und den Debatten um Planung, Prognosen und Futurologie verdankt. Es war das „Max-PlanckInstitut zur Erforschung der Lebensbedingungen der wissenschaftlich-technischen Welt"“. Auch hier gab es schon früh spöttische Bemerkungen, die Lebensbedingungen der wissenschaftlich-technischen Welt seien doch wohl am besten im Ruhrgebiet zu erforschen und nicht im idyllischen Oberbayern. Doch der Direktor des Instituts, Carl Friedrich von Weizsäcker, hätte für einen Umzug nach Castrop-Rauxel wohl nicht seine Professur für Philosophie an der Universität Hamburg verlassen. Und zudem wollte die Max-PlanckGesellschaft das neue Institut in der Nähe ihrer Hauptverwaltung in München ansiedeln. 
30 Mitarbeiter - 1971 wurde die Gruppe um Weizsäcker und seine Philosophen, Physiker und Ökonomen durch Jürgen Habermas als zweiten Direktor und eine weitere Gruppe von noch einmal 15 Soziologen und Philosophen erweitert - versuchten, wie Weizsäcker es selbst einmal genannt hat, ein „Institut für unangenehme Fragestellungen“ aufzubauen. Mehrere Arbeitsgruppen beschäftigten sich mit einzelnen Themenschwerpunkten, die von „Kriegsfolgen und Kriegsverhütung“ über „Ökonomie der Entwicklungsländer“ bis zu „Weltmodellen“" und „Neuen Ideen in der Wissenschaftspolitik“ reichten. Nach einigen Jahren kam auch noch der Bereich „Umweltprobleme und Wachstum“ hinzu.

Und vor dem Hintergrund der 68er Bewegung hatte man auch ganz eigene Vorstellungen darüber, wie die Wissenschaft vorgehen sollte. Michael Drieschner, selbst langjähriger Mitarbeiter am Starnberger Institut, hat es in einem Aufsatz einmal so ausgedrückt:

Die Mitarbeiter wollten die großen Probleme der Welt lösen mit einer ganz neuen Form von Wissenschaft, in der alle gemeinsam gleichberechtigt über alles entscheiden sollten. (Drieschner 2008, S. 13). ${ }^{3}$

Die großen Erwartungen, die die Mitarbeiter des Instituts an ihre eigene Arbeit stellten, konnten sie nicht erfüllen. Und auch der Direktor Weizsäcker verzweifelte zunehmend daran, dass die Öffentlichkeit an den „Überlebensfragen der Menschheit“, an denen das Institut arbeitete, kein großes Interesse zeigte. Mit Weizsäckers Emeritierung 1980 wurde das Institut aufgelöst. Da war der Enthusiasmus für übergreifende Planung und Prognosen bereits weitgehend erloschen - auch innerhalb der Max-Planck-Gesellschaft. Mehrere Mitarbeiter zogen nun in das Kölner Max-Planck-Institut um, das sich unter der Leitung von Fritz Scharpf und Renate Mayntz mit sehr viel konkreteren und beschränkteren Fragestellungen beschäftigte (Mayntz u. Scharpf 1995).

Angesichts der ursprünglich großen Erwartungen an diese Form von Politikberatung und Politikanalyse machte sich bei vielen eine gewisse Enttäuschung breit. Das gilt auch und spezifisch für den Bereich der Wirtschaftspolitik, in dem ab Mitte der 1960er Jahre mit dem „Sachverständigenrat zur Beurteilung der gesamtwirtschaftlichen Lage“ ein Beratungsgremium geschaffen worden war, dass die Bundesregierung in wirtschaftspolitischen Fragen beraten sollte. Aber auch hier ließ sich das Versprechen von der ,Verwissenschaftlichung der Politik“ nicht einhalten. Tim Schanetzky (2007) hat dies unlängst in seiner Frankfurter zeithistorischen Dissertation ausführlich dargestellt und analysiert. Der Titel des Buches gibt bereits den Tenor der Untersuchung wieder: „Die große Ernüchterung“. Schanetzky schildert, wie zunächst in der Großen Koalition mit Karl Schiller die Hoffnung dominiert hatte, wissenschaftliche Grundlagen für Reformpolitik schaffen zu können. Aus einem zunächst im Rahmen des Bundeswirtschaftsministeriums gegründeten „Arbeitskreis Automation“" wurde nach Regierungsantritt der sozialliberalen Koalition 1970 die „Kommission für wirtschaftlichen und sozialen Wandel“ geschaffen. Schiller, der - ähnlich wie Kennedy ein paar Jahre zuvor - davon überzeugt war, die neue Gesellschaft verlange ,technokratisch trainierte Politiker“, war mit den anderen Mitgliedern der Regierung Brandt der Hoffnung, diese Kommission könne zur Überwindung des Mangels an planungsrelevanten Informationen über die wirtschaftlichen und gesellschaftlichen Entwicklungstendenzen beitragen.

3 Zur Geschichte des Instituts siehe ausführlicher auch Drieschner 1996. 
Doch sechs Jahre später, 1977, herrschte bei der Vorlage des 600 Seiten starken Abschlussberichtes der Kommission in der Tat die schon genannte große Ernüchterung. ${ }^{4}$ Nicht nur hatten die mittlerweile eingetretenen weltwirtschaftlichen Verwerfungen im Gefolge der Ölkrisen die politischen Prioritäten verschoben. Es war daneben auch offenkundig, dass man keine eindeutige Quintessenz aus den insgesamt 189 von der Kommission in Auftrag gegebenen Gutachten ziehen konnte. Neben den für Drittmittelantrags- und -abschlussberichtsprosa typischen dehnbaren Leerformeln dominierten vor allem zwei Erkenntnisse die Kommissionsarbeit:

- zum einen, dass strittige Entscheidungen hinsichtlich des gesellschaftlichen und sozialen Wandels auch von Experten nicht eindeutig getroffen werden konnten;

- und zum zweiten wurde klar, dass Planung und Durchsetzbarkeit innerer Reformen zutiefst politische Prozesse waren, denen die Gefahr innewohnte, im Dickicht der Bürokratie stecken zu bleiben.

Diese zentrale Erfahrung wurde auch in Amerika einige Jahre zuvor gemacht. Sie ist vielleicht am besten zusammengefasst im Untertitel eines Buches von zwei führenden Policy-Forschern, Jeffrey Pressman und Aaron Wildavsky, das 1973 erschien und sofort zu einem Klassiker wurde: „How great expectations in Washington are dashed in Oakland, or why it's amazing that federal programs work at all“ - frei übersetzt: ,Wie ursprünglich große Erwartungen in Washington dann vor Ort scheitern, oder warum man sich wundern muss, dass Maßnahmenprogramme der Bundesregierung überhaupt funktionieren“. Der Titel des Buches („Implementation“) war der Startpunkt für einen ganzen neuen Zweig der sozialwissenschaftlichen Politikforschung, nämlich die Implementationsforschung. Hier stand nun im Mittelpunkt, was tatsächlich mit aktivistischer Politik vor Ort passiert und warum die eben schon erwähnten hohen Erwartungen sich oft nicht erfüllen.

Die Implementationsforschung lieferte somit Belege und Beweise dafür, dass die aktivistische Reformpolitik der 1960er Jahre in vielerlei Hinsicht hinter den an sie gestellten Erwartungen zurückblieb. Ohnehin hatte sich, wie schon erwähnt, das politische Klima gewandelt - und mit ihm die politischen Prioritäten. In der Bundesrepublik Deutschland sprach man schon Mitte der 1970er Jahre von einer „Tendenzwende“. Gesellschaftliche und politische Kräfte begannen sich zu formieren, um einen Gegenentwurf zur Reformpolitik der sozialliberalen Koalition politisch durchzusetzen. Diese neue Tendenz, die in der Bundesrepublik mit dem Regierungswechsel von 1982 schließlich politisch erfolgreich war, stellte mehr die Bewahrung des Bestehenden in den Vordergrund und hatte wenige Ansprüche in Bezug auf Reformen. Wie bereits erläutert, ist der Bedarf einer solchen Politik an Politikberatung von wissenschaftlicher Seite eher gering. Aber es wäre zu einfach, eine direkte Gleichsetzung zwischen politischen Richtungen und dem Bedarf nach Politikberatung vorzunehmen - etwa nach der Formel: linke Politik=mehr Beratungsbedarf; rechte Politik= weniger Beratungsbedarf. Beratungsbedarf besteht vor allem, wenn bestehende Verhältnisse verändert werden sollen - und dies kann ebenso das Programm einer politisch eher rechts stehenden Regierung seien.

Ein gutes Beispiel dafür ist die Regierungszeit von Margaret Thatcher in Großbritannien ab 1979. Margaret Thatcher, die sich in der zweiten Hälfte der 1970er Jahre vor

4 Vgl. Bundesminister für Arbeit und Sozialordnung 1977. 
allem unter dem Einfluss ihres Vertrauten Sir Keith Joseph von einer mainstream-Konservativen $\mathrm{zu}$ einer marktliberalen Idealen folgenden Ideologin gewandelt hatte, misstraute dem britischen Beamtenapparat und seiner Reformfreudigkeit sehr (vgl. Busch 1989). Thatcher verdächtigte den civil service, zu tief im korporatistischen Denken der letzten Jahrzehnte verwurzelt zu sein, um ihre fundamentalen Reformpläne tatsächlich umsetzen zu können. Schon mehrere Jahre vor ihrem Regierungsantritt gründete sie deshalb mit Keith Joseph zusammen eine eigene Denkfabrik - einen der ersten Think-Tanks -, die ein in den Vereinigten Staaten bereits wohl etabliertes Modell nach Großbritannien importierte. In diesem Centre for Policy Studies sind viele der Pläne gereift, die nach Thatchers Regierungsantritt in den 1980er Jahren in Großbritannien in Politik umgesetzt wurden und das Land grundlegend verändert haben.

\section{Gründe für die Schwierigkeiten}

Der Bedarf an wissenschaftlicher Politikberatung lässt sich also, das haben wir gesehen, weniger an der politischen Ausrichtung einer Regierung festmachen als vielmehr am Ausmaß des Wandels, den man in der Politik plant. Dennoch ist es ganz eindeutig, dass die Popularität und der Bedarf nach Politikberatung über die Zeit erheblichen Schwankungen unterliegen. Der Euphorie für Politikberatung und „Verwissenschaftlichung von Politik“ in den 1960er Jahren folgte in vielen Ländern eine Phase der Enttäuschung. ${ }^{5}$

Lassen Sie mich deshalb im Folgenden einige Gründe anführen, warum das Verhältnis zwischen politischer Praxis und wissenschaftlicher Beratung oft ein so schwieriges ist. Wie wir sehen werden, gibt es dabei Hemmnisse sowohl von Seiten der Wissenschaftler als auch von Seiten der Politik. Und auch in der Öffentlichkeit unterliegt das Ansehen wissenschaftlicher Expertise in der Politikberatung Schwankungen: Hatte man in den sechziger Jahren den Experten noch großes Vertrauen entgegengebracht, so dominierten in den siebziger und achtziger Jahren Gefühle von Ernüchterung und Enttäuschung. Die Erfahrungen mit verschiedenen Expertengremien hatten deutlich gemacht, dass es auch in ihnen zu einer Politisierung kommen konnte, und insbesondere der Konflikt um die Nutzung der Atomenergie zeigte, dass es auch für gegensätzliche Positionen Experten geben konnte, die diese jeweils befürworteten. Der gerade in Deutschland populäre Traum, schwierige und konfliktuöse Entscheidungen einfach an Experten und Sachverständige delegieren zu können, löste sich in Luft auf.

Aber auch seitens der Wissenschaft gab es Skepsis gegenüber den Beratungen der Praxis - beziehungsweise, wie man es eher auszudrücken pflegte, der „Macht“. Den Widerstand auch etablierter Wissenschaftsorganisationen gegen die Gründung des WZB im Jahr 1969 habe ich bereits angeführt. Er war durchaus typisch für eine Haltung in weiten Teilen der Sozialwissenschaften, die wissenschaftlicher Politikberatung unter den Verdacht von Technokratie stellte, die einer Demokratie nicht angemessen sei. Dabei folgte

5 Instruktiv ist etwa die Reflexion Carl Friedrich von Weizsäckers über die Rolle des weiter oben diskutierten Max-Planck-Instituts: „Unser Institut hat Analysen gegeben, engagierte, heterogene, unabgeschlossene. Was mir gefehlt hat, war die breite Konkurrenz. Wir waren in der Wissenschaft unseres Landes ein wenig allein.“ (von Weizsäcker 1983, S. 344) 
man einer Unterscheidung von Beratungsmodellen, wie sie zuerst Jürgen Habermas 1963 in seinem Aufsatz „Verwissenschaftlichte Politik und öffentliche Meinung“ aufgestellt hatte (Habermas 1968). Gerade die zur Theoriefixierung neigenden deutschen Sozialwissenschaftler tendierten dazu, wissenschaftliche Politikberatung entweder als Feigenblatt für den politischen Betrieb zu sehen (wofür man sich nicht hergeben wollte), oder sie als technokratische Beherrschung zu interpretieren (was ebenfalls als nicht hinnehmbar angesehen wurde). Dass Konservative wie der Soziologe Helmut Schelsky bereits zu Beginn der 1960er Jahre von einem „Ende der Ideologien“ zu Gunsten einer „Herrschaft der Experten“" gesprochen hatten (vgl. Schelsky 1961, S. 20 ff.), machte es für die sich weitgehend als links verstehenden Sozialwissenschaften nicht einfacher, sich pragmatisch auf eine Beratungstätigkeit einzulassen, wie das in vielen anderen Fächern durchaus möglich war.

Das verbreitete Selbstverständnis der Politikwissenschaft als „Oppositionswissenschaft" führte dazu, dass die tatsächlich existierenden Gremien in der Bundesrepublik von Juristen und Ökonomen dominiert wurden und man sich gewissermaßen selbst ins Abseits stellte. Dass die Rigidität des deutschen Beamtenrechts einen personellen Austausch auf Zeit zwischen Hochschule und Verwaltung fast unmöglich machte, kam erschwerend hinzu. Wir können also festhalten, dass die deutschen Politikwissenschaftler - von wenigen Ausnahmen, vor allem im Bereich Außenpolitik, abgesehen, wie etwa Karl Kaiser oder Peter Christian Ludz - nicht gerade danach drängten, die politische Praxis zu beraten.

Aber auch auf Seiten der Politik gibt es Faktoren, die Schwierigkeiten mit der Politikberatung erklären können. Natürlich möchten politische Akteure beraten werden - jedenfalls von Zeit zu Zeit, nämlich dann, wenn sie nicht wissen, welche Lösungen sie anstreben sollten. Aber das heißt auch: Politische Akteure möchten am liebsten punktuell beraten werden. Formen institutionalisierter permanenter Beratung können hingegen auch auf das Desinteresse von politischen Akteuren treffen, nämlich deshalb, weil sie deren politischen Entscheidungsspielraum einschränken. Experten können sich ja selbst in die Rolle politischer Akteure begeben, und wenn sie dann mit Legitimität gegenüber der Öffentlichkeit agieren, können sie durch ihre Äußerungen den Handlungsspielraum der politischen Akteure erheblich reduzieren. Gerade in der expertengläubigen deutschen politischen Kultur haben Politiker solche Befürchtungen gehegt - zumindest in der Vergangenheit: So ist es etwa im aus dem Jahr 1963 stammenden Gesetz über den Sachverständigenrat zur Begutachtung der gesamtwirtschaftlichen Lage den Mitgliedern des Sachverständigenrates laut $\S 2$ verboten, ,Empfehlungen für bestimmte wirtschaftsund sozialpolitische Maßnahmen [auszusprechen]“ - man befürchtete wohl, der Regierung bliebe dann nichts anderes übrig, als diesen Ratschlägen zu folgen. Und neben der Exekutive kann natürlich auch der Handlungsspielraum der Legislative durch Beratung eingeschränkt werden - denn trotz oft langjähriger Debatten (z. B. unter dem Stichwort „Technikfolgenabschätzung“) sind ja in den meisten Ländern die Versuche, den Parlamenten genuin eigene Beratungskapazitäten zu schaffen, nicht sehr weit gediehen - auch in Deutschland.

Zusammenfassend können wir also sagen, dass das Interesse der verschiedenen Akteure im politischen System an Beratung durch auswärtigen Sachverstand durchaus signifikant variieren kann und dass wir hierin sowohl einen Hauptgrund für die unterschiedliche 
Nutzung bisher wie auch eine zu berücksichtigende Variable bei Plänen für Ausweitung von Politikberatung erkennen können.

\section{Schluss}

Lassen Sie mich nun zum Schluss kommen mit der Frage, welche verschiedenen Funktionen wissenschaftliche Beratung eigentlich für das politische System erfüllen kann. Ich greife dabei zurück auf eine Typologie, die Klaus von Beyme (1988) in einem Aufsatz vor mehr als 20 Jahren vorgelegt hat, und in der er vier unterschiedliche Funktionen nennt:

- die Funktion als Frühwarnsystem und Problemerkennungsmechanismus für die Regierenden;

- die Schlichtungsfunktion für staatliche Agenturen;

- die Kontroll- und Überwachungsfunktion, und schließlich

- die Legitimationsfunktion für bereits getroffene Entscheidungen.

Am unproblematischsten erscheint dabei die Problemerkennungsfunktion - sie ist genuiner Gebrauch wissenschaftlicher Kompetenz, auch wenn der Staat Probleme haben mag, die Ergebnisse schließlich angemessen zu verarbeiten. Die bereits genannte „Kommission für wirtschaftlichen und sozialen Wandel" dient auch von Beyme als Beispiel für ein Unternehmen, das letztlich eigentlich mehr der Wissenschaft als den Auftraggebern diente. Ein Nachteil von Politikberatung in diesem Bereich ist vielleicht, dass es zu Ungleichgewichten zwischen Exekutive und Legislative kommt - wie eben schon angesprochen. Daneben und hauptsächlich stellt sich aber die Frage, wie Kapazität zur Beratung organisiert werden soll.

Die Konfliktschlichtungs- und Kontrollfunktionen erscheinen ebenfalls unproblematisch und sind wohl nur selten politisch umstritten; das gilt jedoch nicht für die sicher problematischste Form des Gebrauchs von externem Sachverstand in der Politik, nämlich die der Legitimation von bereits getroffenen Entscheidungen. Man sollte sie deshalb vielleicht besser „Feigenblattfunktion“ nennen und sie als im negativen Sinn politische Funktion interpretieren. Ich denke, Politikberatung, die das Adjektiv wissenschaftlich zu Recht weiter tragen möchte, sollte sich für solche Funktionen nicht hergeben; das sollte man den Experten aus dem Bereich public relations überlassen - und genau hinschauen, wenn sich professionelle Berater unter dem Label „public affairs“ faktisch zu spin doctors der Politik machen.

Dabei ist die Grenze oft kaum genau zu bestimmen. Der vor allem in Großbritannien unter der Blair-Regierung populäre Trend zum evidence-based policy making ist ja im Prinzip durchaus zu begrüßen (Young et al. 2002). Aber man sollte sich eben auch der Gefahr bewusst sein, dass die Auftraggeber eventuell Interesse an einem policy-based evidence making haben - und dazu sollte sich, so denke ich, Wissenschaft nicht hergeben. Aber vielleicht überschätze ich hier auch die moralische Selbststeuerungsfähigkeit der Wissenschaft in der heutigen Zeit, zumal im Angesicht von an persönliche Bezahlung gebundenen sogenannten ,Zielvereinbarungen“ mit Universitätsleitungen...

Zusammenfassend kann man festhalten, dass wissenschaftliche Beratung wichtige Funktionen für das politische System erfüllen kann, die praktische Umsetzung aber 
bereits angesprochene Kapazitäts- und Organisationsfragen aufwirft. Im akademischen System kann umfangreiche Beratungskapazität nicht ,auf Verdacht“" vorgehalten werden; das war schon in Zeiten der Hochschulexpansion nicht möglich und ist es heute noch viel weniger: Dafür sind die Mäntel der Kapazitätsverordnung zu eng geschneidert. Gliedert man die Beratungskapazität aber aus den Universitäten aus, etwa in eigene Institute, läuft man Gefahr,

- entweder die Verbindung zur Wissenschaft (und ihrer Kreativität) durch Bürokratisierung zu verlieren (und damit gerade jene Außenperspektive auf das politisch-administrative System, die externe Beratung erst wertvoll macht)

- oder autonome Spielwiesen nach Art des Starnberger MPI zu schaffen, die sich in teutonischer Tradition lieber um die großen Weltzusammenhänge kümmern als um die konkreten Entscheidungen der alltäglichen Politik und die Zuarbeit für die Ministerialbürokratie.

Man muss nicht einem Luhmann'schen Steuerungspessimismus huldigen, um hier zur Diagnose zu kommen, dass ein Miteinander und ein konstruktiver Dialog zwischen den beiden gesellschaftlichen Teilsystemen Politik und Wissenschaft besonders schwierig sind. $\mathrm{Ob}$ die gegenwärtige Krise so tief sein wird, dass sich an diesen Umständen etwas ändern wird, darüber wage ich keine Prognose; wünschen möchte man es sich eigentlich kaum.

So bleibt denn zum Abschluss nur der (etwas hilflos klingende, aber pragmatisch gemeinte) Appell an die Wissenschaftler, sich den ,öffentlichen Dingen“, den ,,res publicae“, nicht nur nicht zu verweigern, sondern aus eigener Verantwortung zu ihnen beizutragen, ohne dabei ihre spezifische Distanz und Perspektive zu verlieren. Auch so kann Beratungskapazität geschaffen werden.

Klaus von Beyme, so scheint mir, hat diese Perspektive als Politikwissenschaftler immer eingenommen. Er hat seinen Rat nicht versagt und sich dort zu Wort gemeldet, wo er Wichtiges beizutragen hatte. Er ist, auch in dieser Hinsicht, ein Beispiel, an dem wir uns orientieren können.

Open Access: Dieser Artikel unterliegt den Bedingungen der Creative Commons Attribution Noncommercial License. Dadurch sind die nichtkommerzielle Nutzung, Verteilung und Reproduktion erlaubt, sofern der/die Originalautor/en und die Quelle angegeben sind.

\section{Literatur}

Akerlof, G., \& Shiller, R. (2009). Animal spirits. How human psychology drives the economy, and why it matters for global capitalism. Princeton, N.J.: Princeton University Press.

von Beyme, K. (1988). Politik und wissenschaftliche Information der Politiker in modernen Industriegesellschaften. In K. von Beyme (Hrsg.), Der Vergleich in der Politikwissenschaft (S. 347-368). München: Piper.

von Beyme, K. (2009). Vorläufer der Politikfeldanalyse auf dem europäischen Kontinent. In K. Schubert \& N. C. Bandelow (Hrsg.), Lehrbuch der Politikfeldanalyse (S. 25-36). München: Oldenbourg.

Bleek, W. (2001). Geschichte der Politikwissenschaft in Deutschland. München: Beck.

Bryce, J. (1921). Modern democracies. New York: The Macmillan Company. 
Bundesminister für Arbeit und Sozialordnung (Hrsg.). (1977). Wirtschaftlicher und sozialer Wandel in der Bundesrepublik Deutschland. Gutachten der Kommission für Wirtschaftlichen und Sozialen Wandel. Göttingen: Schwartz.

Busch, A. (1989). Neokonservative Wirtschaftspolitik in Großbritannien. Vorgeschichte, Problemdiagnose, Ziele und Ergebnisse des ,,Thatcherismus “. Frankfurt a.M.: Lang.

Dahrendorf, R. (1965). Gesellschaft und Demokratie in Deutschland. München: Piper.

Drieschner, M. (2008). Obituary: Carl Friedrich von Weizsäcker. Journal for General Philosophy of Science, 39(1), 1-16.

Finer, H. (1932). The theory and practice of modern government. London: Methuen.

Friedrich, C. J. (1937). Constitutional government and democracy. Theory and practice in Europe and America. Boston: Little, Brown and Company.

Habermas, J. (1968). Verwissenschaftlichte Politik und öffentliche Meinung. In J. Habermas (Hrsg.), Technik und Wissenschaft als „Ideologie“(S. 120-145). Frankfurt a.M.: Suhrkamp.

Heclo, H. (1974). Modern social politics in Britain and Sweden. From relief to income maintenance. New Haven: Yale University Press.

Kraus, H.-C. (2004). Vorformen und Anfänge wissenschaftlicher Politikberatung im 19. Jahrhundert. In S. Fisch \& W. Rudloff (Hrsg.), Experten und Politik. Wissenschaftliche Politikberatung in geschichtlicher Perspektive (S. 59-78). Berlin: Duncker \& Humblot.

Lerner, D., \& Lasswell, H. D. (1951). The policy sciences. Recent developments in scope and method. Stanford: Stanford University Press.

Mayntz, R. \& Scharpf, F. W. (1995). Gesellschaftliche Selbstregelung und politische Steuerung. Frankfurt a.M.: Campus.

Münkler, H. (1982). Machiavelli. Die Begründung des politischen Denkens der Neuzeit aus der Krise der Republik Florenz. Frankfurt a.M.: Europäische Verlagsanstalt.

Pressman, J. L., \& Wildavsky, A. B. (1973). Implementation. How great expectations in Washington are dashed in Oakland. Or, why it's amazing that federal programs work at all, this being a saga of the economic development administration as told by two sympathetic observers who seek to build morals on a foundation of ruined hopes. Berkeley: University of California Press.

Schanetzky, T. (2007). Die große Ernüchterung. Wirtschaftspolitik, Expertise und Gesellschaft in der Bundesrepublik 1966 bis 1982. Berlin: Akademie Verlag.

Schelsky, H. (1961). Der Mensch in der wissenschaftlichen Zivilisation. Köln: Westdeutscher Verlag.

Stoop, P. (2009). Der kurze Frühling der Empörung. Erst ein Jahr nach der Gründung entbrannte 1970 der öffentliche Streit ums WZB. WZB-Mitteilungen, 123, 58-59.

von Weizsäcker, C. F. (1983). Rechenschaft vor der Öffentlichkeit. Als Physiker zwischen Philosophie und Politik. In C. F. v. Weizsäcker (Hrsg.), Wahrnehmung der Neuzeit (S. 329-348). München: Hanser.

Young, K., Ashby, D., Boaz, A., Grayson, L. (2002). Social science and the evidence-based policy movement. Social Policy and Society, 1, 215-224. 


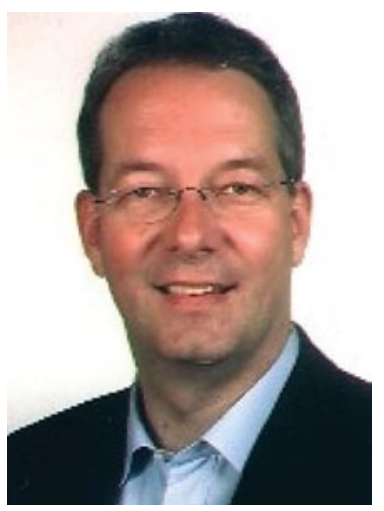

Prof. Dr. Andreas Busch ist Geschäftsführender Direktor des Seminars für Politikwissenschaft an der Georg-August-Universität Göttingen und Inhaber des Lehrstuhls für Vergleichende Politikwissenschaft und Politische Ökonomie. Sein Arbeitsschwerpunkt ist die Vergleichende Staatstätigkeitsforschung, insbesondere in den Bereichen Regulierung, Wrtschafts- und Informationspolitik. Seine letzte Veröffentlichung ist das Buch „Banking Regulation and Globalization“ (Oxford University Press 2009). 